\title{
Particular evolutive modalities in SARS-CoV-2 infection
}

\author{
Stefan Lazari, ${ }^{1,2}$, Adela Neculai', Loredana Musetescu' ${ }^{2}$ Carmen Marcu², \\ Simina Dinu' ${ }^{2}$, Maria Nica ${ }^{1,2}$, Emanoil Ceausu ${ }^{1,2,3}$, Simin-Aysel Florescu ${ }^{1,2}$ \\ 1"Carol Davila" University of Medicine and Pharmacy, Bucharest, Romania \\ 2"Dr. Victor Babes" Clinical Hospital of Infectious and Tropical Diseases, Bucharest, Romania \\ ${ }^{3}$ Academy of Medical Sciences of Romania
}

\begin{abstract}
The COVID-19 pandemic has challenged the medical world in many ways regarding diagnosis troubles and therapeutic options. In this paper, we will present three cases of patients from different age groups, in which we identified specific ways of evolution, from both clinically and laboratory data point of view. We used a critical evolution risk estimation calculator at admission and compared the results with the subsequent evolution of patients. From the analysis of the presented cases, we found persistent organic damage that will require their long-term follow-up.
\end{abstract}

Keywords: SARS-CoV-2, COVID-19, admission risk calculator, Tocilizumab, RT-PCR, polycythemia

\section{INTRODUCTION}

With the spread of the COVID-19 pandemic, the medical world has faced multiple challenges in terms of diagnosis and treatment options. Numerous reports and studies have been published, which have succeeded in drawing evolutionary patterns in patients infected with SARS-CoV-2, useful in making rapid therapeutic decisions. In this regard, studies showing predictive factors of unfavorable evolution [1,2] or scores trying to estimate the risk of serious evolution and admission to intensive care $[3,4]$ have emerged. In this paper, we (retrospectively) used a patient admission risk calculator, developed by Guangzhou University, which takes into account 10 parameters: age, chest X-ray abnormalities, dyspnea, hemoptysis, consciousness, cancer history, other comorbidities, lactate dehydrogenase $(\mathrm{LDH})$, bilirubin and the ratio of neutrophils to lymphocytes (N/L) [3].

\section{CASES PRESENTATION}

\section{Case 1}

A 35-year-old man, doctor working for the Suceava Ambulance, presents on his own initiative at our clinic on 28.03.2020, with an intensely altered general condition, fever (up to $40^{\circ} \mathrm{C}$ ), chills, myalgias, dyspnea, dry cough, phenomena progressively accentuated in 72 hours. During the last 24 hours, the symptoms become associated with nausea, loss of appetite, diarrhea. The patient was overweight, with a body mass index (BMI) of $28.3 \mathrm{~kg} / \mathrm{m} 3$ and had no known significant pathological history.

From the epidemiological investigation, we didn't retain any known contact of the patient infected with SARS-CoV-2, but, by the nature of the profession, this was plausible. In addition, the patient came from the largest outbreak of COVID-19 recorded in Roma- 
nia (Suceava), in a period of exponential growth in the number of cases of infection [5].

At admission, the patient had a mediocre general condition, generalized myalgias, diarrhea, was afebrile, tachycardic, tachypneic, with dyspnea accentuated by moderate exertion, having $\mathrm{SaO} 2=97 \%$. Given the epidemiological context and symptoms of the patient present at hospitalization, the suspicion of SARS-CoV-2 was raised and the treatment with Hydroxychloroquine and Azithromycin was initiated from the moment of admission, in conformity with the hospital's internal protocol, the infection being confirmed after approximately 6 hours from the admission. At that time, pulmonary radiography showed an intense accentuation of the bilateral infrahilar peribronhovascular interstitial pattern and a lamellar atelectasis in the right middle lung field. According to the therapeutic protocol in force at that time, the treatment was supplemented with lopinavir/ritonavir.

Using the risk calculator for the critical evolution of the patient at admission [3], we established a score of 121.1 points, corresponding to an average degree of risk [0.2372 (95\% CI: 0.1732-0.3159)].

In the first 3 days of hospitalization, the evolution was progressively unfavorable, by aggravation of dyspnea, persistence of fever, digestive phenomena (accentuated by the administration of oral medication), on the 4th day the patient was admitted to intensive care, due to marked respiratory damage $(\mathrm{SaO} 2=$ $84 \%$ under oxygen therapy). Here, he received treatment with Tocilizumab (800 mg/administration), 3 administrations, Dexamethasone (16 mg/day), Meropenem, oxygen therapy was maintained with flows of 10-12 $1 / \mathrm{min}$, the evolution being slowly favorable. After 4 days of hospitalization in ICU, the patient is readmitted to the infectious diseases department, the evolution being slowly favorable. Gastric intolerance and vomiting persisted, despite therapy, for another 4 days. On the 11th day of hospitalization, the patient no longer needed oxygen therapy. He was discharged after 14 days of hospitalization with satisfactory oxygen saturations (95-96\%), good condition, asymptomatic, with two negative SARS-COV 2 RT-PCR tests and having positive IgG SARS-CoV-2 antibodies.

In the figure 1 we will present the evolution of the main laboratory parameters, but also the radiological evolution of the patient during hospitalization.

The figure 1 shows a marked decrease in lymphocytes and monocytes, correlated with a marked increase in inflammatory syndrome and worsening of the general condition on the 6th day of illness. Consistent with these, we found a marked increase in LDH values (from 240 to $455 \mathrm{IU} / \mathrm{l}$ ) and, especially, creatine kinase (2,703 IU/1). The neutrophil/ lymphocyte ratio, considered in the literature as an important indicator of aggravation [1], had a value of 2.1. The patient did not have altered levels of troponin, d-dimers, procalcitonin or serum transaminases. Pulmonary, the radiological aspect showed an obvious aggravation, from the presence of an intense accentuation of the bilateral peribronhovascular pattern (at hospitalization) to the appearance of diffuse alveolo-interstitial infiltrates distributed on the right and left infrahilar regions (Fig. 2).

After discharge from ICU, consistent with the clinical improvement, we found the normalization of lymphocytes rate, with the appearance of monocytosis. Moreover, the calculation of the Pearson coefficient $(0.812386)$ showed an important correlation between these two parameters throughout the hospitalization period. In the analysis of the data in dynamics, we did not take into account the dynamics of the neutrophil/lymphocyte ratio, as the level of neutrophils was altered by the cortisone treatment ad-

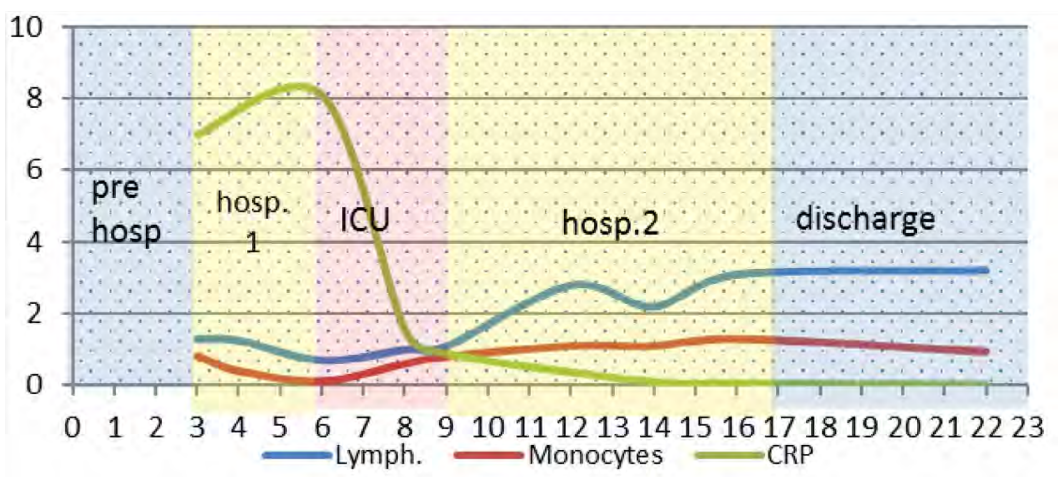

FIGURE 1. Evolution of patient 1, according to haematological and inflammatory parameters 
ministered. After reaching a maximum on the 9th day of evolution (738 U/1), the LDH value progressively decreased to $447 \mathrm{U} / 1$ at discharge on the 16th day. Throughout the hospitalization, the values of d-dimers and troponin remained at normal levels. The patient showed a progressive increase in TGP values (up to 6 times at the time of discharge), as a consequence of Tocilizumab administration. The radiological aspect at discharge shows the substantial improvement of the lesions described above, with the reduction in size and intensity of the right basal alveolar condensation process, with the maintenance of interstitial infiltrates, distributed bilaterally, more importantly in the periphery of lungs (Fig. 3).

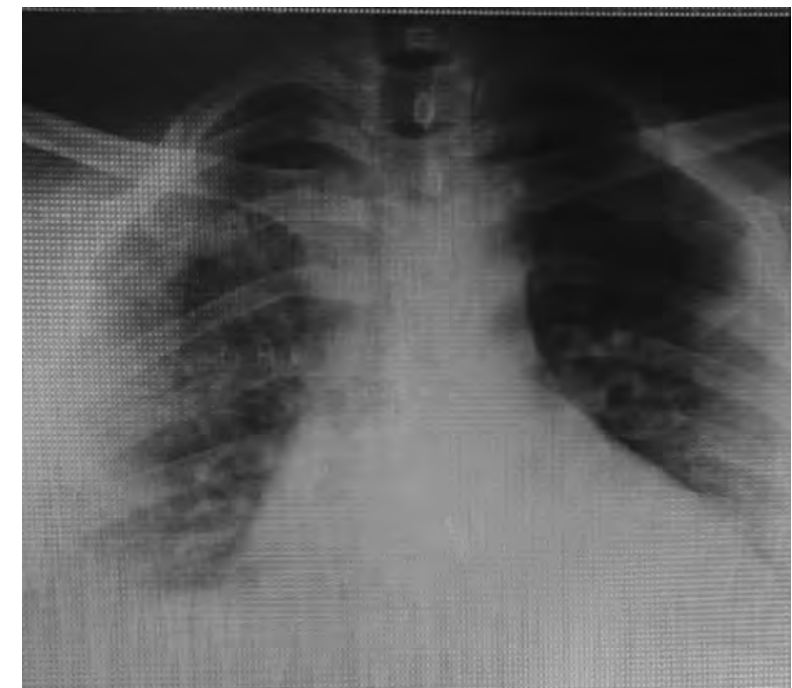

FIGURE 2. Radiological appearance on day 7 of evolution (patient 1)

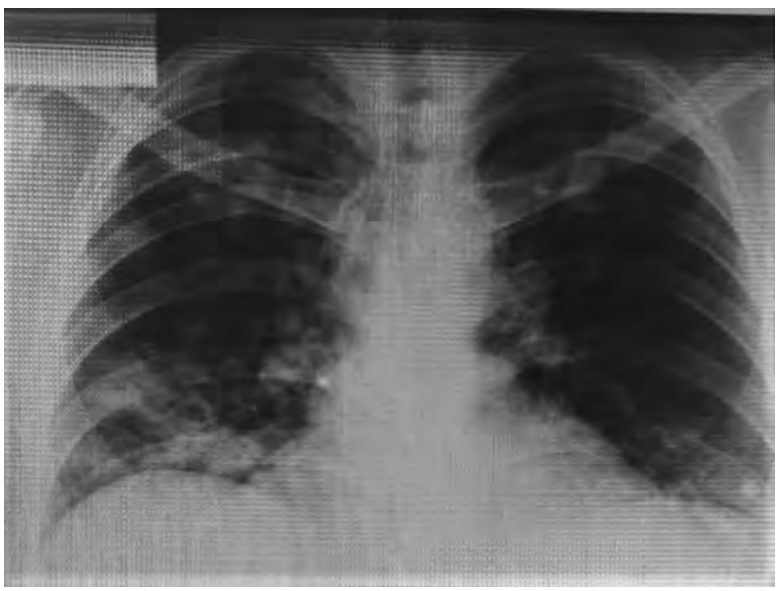

FIGURE 3. Radiological appearance on day 16 of evolution (patient 1)

After 6 days from the hospital discharge, the patient complains of severe chest stabbing and has an episode of hemoptysis, which is why paraclinical investigations are performed, including chest CT. Res- piratory function tests described significant restrictive dysfunction, with an estimated lung age of 78 years [6]. The biochemical analysis showed the normalization of the hemogram values, the improvement of the TGP value (130 U/l). Pulmonary tomography showed changes with chronic potential (Fig. 4).
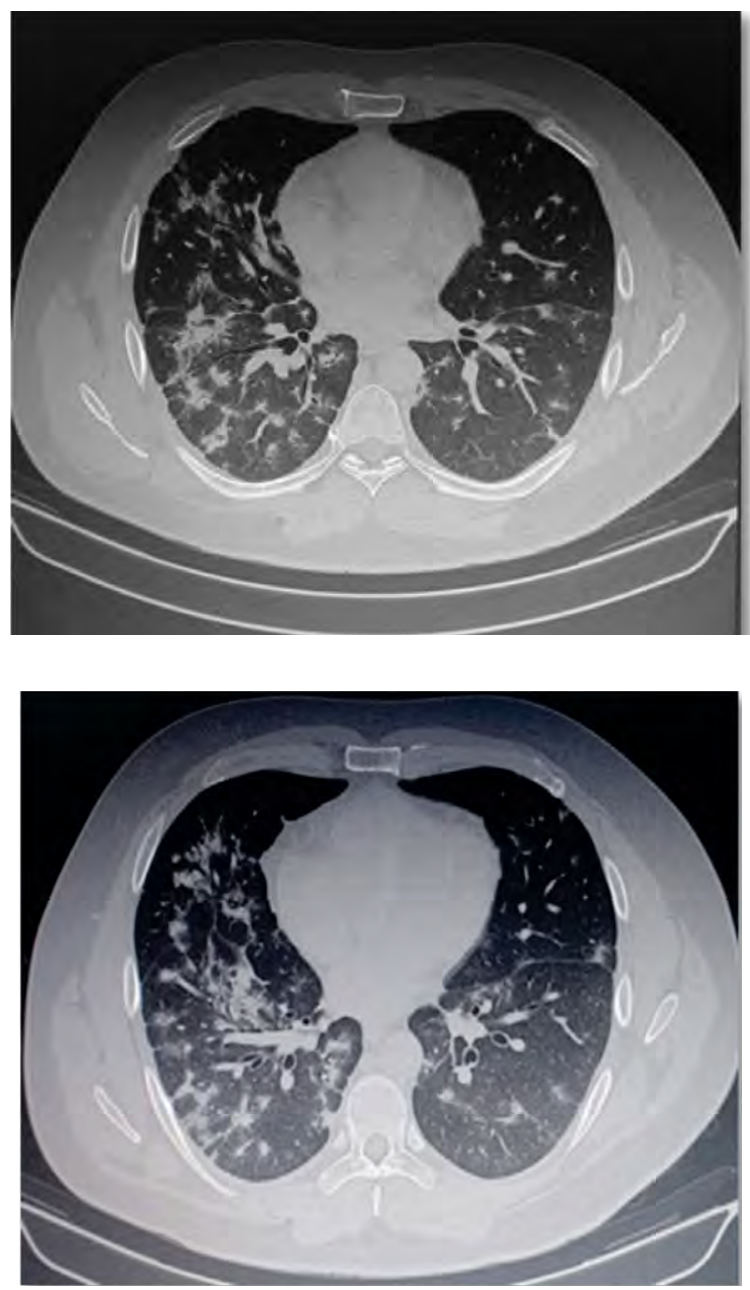

FIGURE 4. Aspect of pulmonary CT on day 22 from the onset of the disease. Ground glass opacities, with subpleural/ peripheral distribution, some with a tendency to form focal condensation. Scattered, irregularly shaped focal condensations, with a tendency to form fibrotic plaques, some containing traction bronchiectasis.

\section{DISCUSSIONS}

The case presented above showed a severe evolution, with a major risk of subsequent chronicity in a non-smoking patient, without significant risk factors or history. If at the time of hospitalization there were no serious factors, subsequent evaluations detected the appearance of some phenomena during days 5 and 6 of evolution the appearance of some phenomena, which were correlated with the appearance of a "cytokine storm", even if, the determination of the value 
IL-6 within the clinic was not possible at the given time. Thus, in the case of this patient, the sudden decrease of lymphocyte, monocyte values, correlated with the increase of CRP, LDH values and the aggravation of the radiological aspect could estimate the appearance of this moment in the evolution of the disease. Also, in a patient who has had a severe evolution, the virological cure does not always coincide with the clinical cure, requiring a medium or longterm follow-up, to find the occurrence of phenomena that can irreversibly affect the subsequent evolution

\section{Case 2}

58-year-old patient is brought by the Ambulance service to our clinic for symptoms started 5 days ago, with asthenia, fever (maximum $\mathrm{T}=38.5^{\circ} \mathrm{C}$ ), dry cough, myalgia, intermittent dyspnea (several episodes on day). The patient had no known epidemiological link.

From the pathological antecedents, we noticed an episode of electrocution (2017), when he was diagnosed with hypertension, for which he received chronic treatment. He was a former smoker (ceased for 20 years) and an occasional drinker. At the clinical examination at hospitalization, we found grade 2 obesity $\left(\mathrm{BMI}=36.2 \mathrm{~kg} / \mathrm{m}^{3}\right)$, mild hepatomegaly and elevated arterial pressure values $(170 / 90 \mathrm{~mm} \mathrm{Hg})$, the rest of the clinical examination being within normal limits.

At the time of admission, the patient was not dyspneic and had $\mathrm{SaO} 2=97 \%$ in atmospheric air. Paraclinically, at hospitalization, we found moderate lymphopenia $\left(900 / \mathrm{mm}^{3}\right)$, inflammatory syndrome (increased CRP and fibrinogen, but with normal procalcitonin values), signs of cellular lysis (LDH, CK with double and triple values, respectively, compared to the maximum limit of normal ), increase in the level of d-dimers. Pulmonary radiography suggested a characteristic appearance for SARS-CoV-2 infection, with mixed interstitial-alveolar infiltrates extending to both lung fields, especially in the periphery (Fig. 5).

Using the admission risk calculator [3], we found that the patient had a high risk (167.7 points), with a probability of 0.7167 (95\% CI: 0.6301-0.7897).

Due to the suggestive clinical, biological and imaging aspect, the patient received treatment consisting of Hydroxychloroquine, Azithromycin, according to the local clinic protocol, without waiting for the RT-
PCR SARS-CoV-2 result. In fact, of the 3 nasopharyngeal exudate samples collected after hospitalization at 24-hour intervals, the presence of the virus could not be detected.

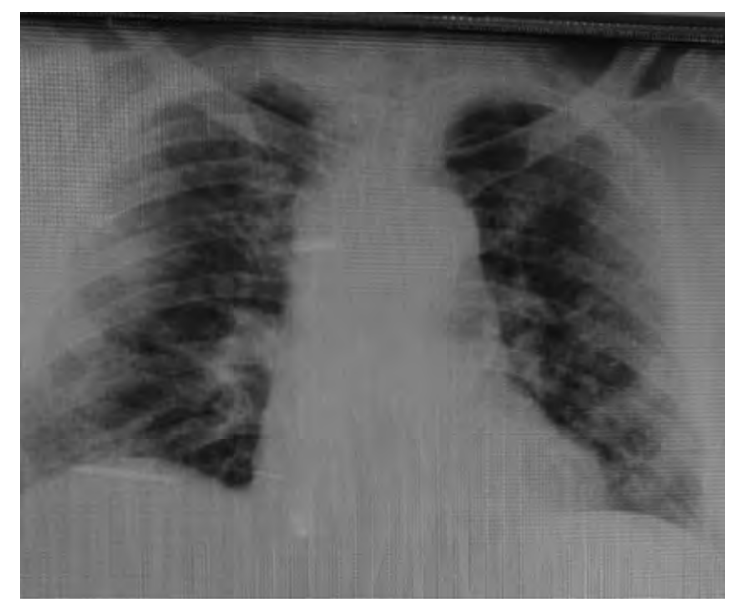

FIGURE 5. Radiological appearance on day 5 of disease progression (patient 2)

Upper tract respiratory panel (QIAGEN Multiplex PCR) was performed by sputum, with negative results. In the first 24 hours after hospitalization, the patient showed an unfavorable evolution, with the appearance of tachypnea, decrease of $\mathrm{SaO} 2$ to levels of $80-82 \%$ and worsening of the radiological aspect, reason for which the patient received two administrations of Tocilizumab of $800 \mathrm{mg}$ each. On the 8 th day of evolution we highlighted a maximum of the CRP value $(4.56 \mathrm{mg} / \mathrm{dl})$, a maximum of the $\mathrm{N} / \mathrm{L}$ ratio $(9.66)$ and the presence of an increased value of IL-6 (23.99 $\mathrm{pg} / \mathrm{ml}$ ). From the biological data, we noted the existence of an important correlation between the values of the neutrophil / lymphocyte ratio (N/L) and of the CRP with the clinical evolution of the patient. In fact, the variation of the two parameters showed an important correlation (Pearson coefficient $=0.903$ ) throughout the hospitalization.

The patient was discharged on the 15th day of evolution (10th day of hospitalization) in good general condition and asymptomatic, the pulmonary radiological aspect finding a substantial improvement. It showed 2-fold increased serum transaminases (probably a consequence of Tocilizumab), but with a nearnormal decrease in LDH. In addition, a rapid antibody test was performed at discharge and the presence of IgG-SARS-CoV-2 antibodies was found. That result could support the retrospective diagnosis of the infection. 


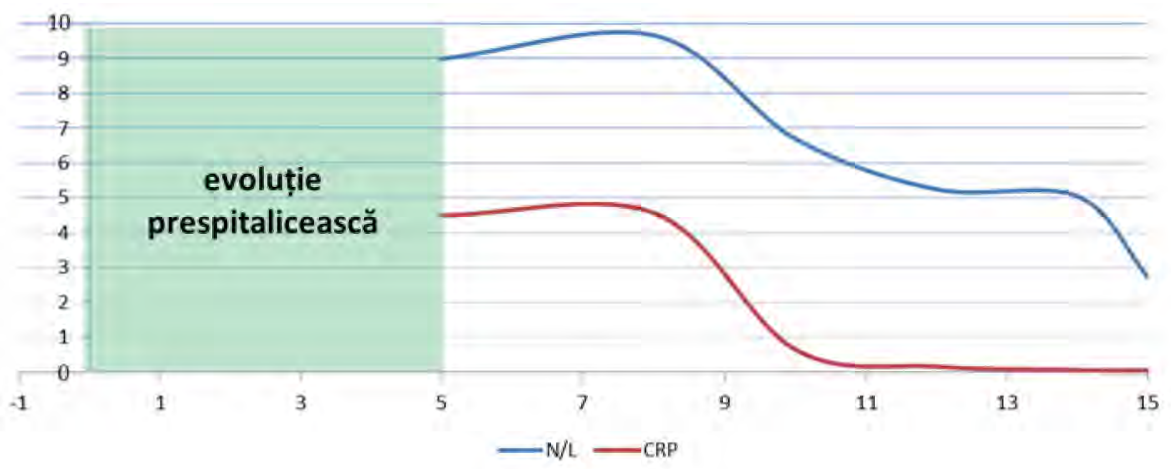

FIGURE 6. Evolution of the neutrophil / lymphocyte ratio vs CRP (patient 2)

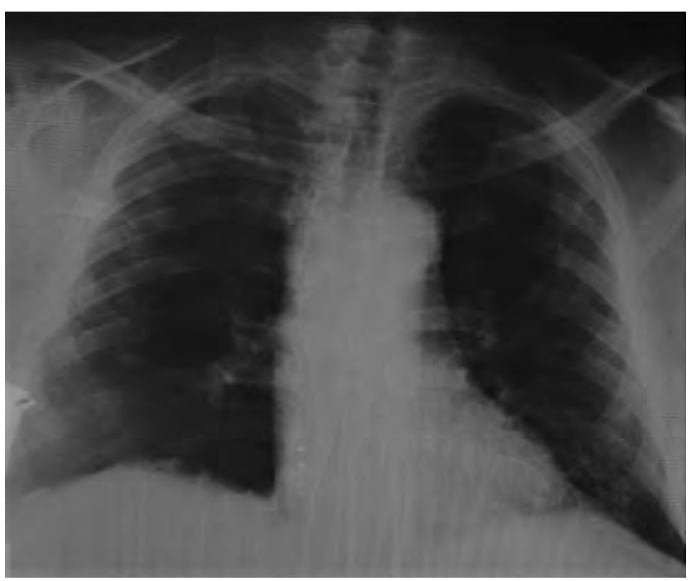

FIGURE 7. Radiological appearance on day 15 of disease progression (patient 2)

\section{DISCUSSIONS}

The described patient, despite not meeting the virological criteria, received the diagnosis of COVID-19, based on both clinical and paraclinical data that we considered suggestive, according to data from the literature [7-9]. Thus, in favor of the positive diagnosis, they pleaded the clinical data and the evolution of the patient, the modification of the biological data, the suggestive radiological aspect. In addition, administration of two doses of Tocilizumab on the 8th day of evolution led to a rapid amendment of symptomatology and an improvement in hematological and biochemical parameters. Unfortunately, due to the epidemiological situation at that time, the patient could not be followed up.

\section{Case 3}

72-year-old patient, previously confirmed with SARS-CoV-2 infection, is brought to our clinic for headaches, vomiting, myalgias, diarrhea, symptoms with onset of about 2 weeks. The patient had no known epidemiological link and from the anamnesis we could not retain previous conditions. Clinically, at admission, the patient was slightly underweight (BMI $=20.2 \mathrm{~kg} / \mathrm{m} 3)$, had dry mucous membranes and cold skin, scleral subicterus, was tachycardic (110 bp/min), the rest of the clinical examination being within normal limits.

Hematologically, there was a significant polycythemia $(\mathrm{Hb}=21.4, \mathrm{Ht}=66 \%)$ leukocytosis with significant neutrophilia and lymphopenia $(\mathrm{N} / \mathrm{L}$ ratio $=$ 26.6). Biochemically, we found liver damage with moderate increases in TGP, bilirubin and GGT, renal failure (creatinine $=3.5 \mathrm{mg} / \mathrm{dl}$ ) and hypernatremia. The pulmonary radiograph performed did not show specific images of COVID-19, although, at the time of hospitalization, the patient had episodes of desaturation $(\mathrm{SaO} 2=88-90 \%)$.

Calculating the risk score at the hospital admission [3], we found that the patient had 160.8 points, with a probability of 0.6493 (95\% CI: $0.5550-0.7333$ ), which represents a high risk of severe evolution.

Since hospitalization, the patient has received treatment according to the local protocol (Hydroxychloroquine, Azithromycin, anticoagulant in therapeutic doses). Considering to be an important dehydration syndrome, the patient was infused i.v., obtaining a significant decrease in creatinine, but with the maintenance of polyglobulia and significant hypernatremia. The patient's evolution was unfavorable in the first days of hospitalization, with a significant increase in the $\mathrm{N} / \mathrm{L}$ ratio (34.2) and IL-6 (16.7 pg/ml), receiving a dose of Tocilizumab $(8 \mathrm{mg} / \mathrm{kg})$.

Due to the persistence of polycythemia, the patient was transferred to the hematology clinic for investigations and a phlebotomy of necessity.

The subsequent evolution of the patient was good, with the progressive decrease of hemoglobin values, $\mathrm{N} / \mathrm{L}$ ratio, the patient being discharged even with mild 
anemia and normal leukocyte values. During hospitalization, we found an increase in serum transaminases (possibly a consequence of Tocilizumab treatment) and normalization of creatinine. Interestingly, hypernatremia was maintained in the first part of hospitalization, consistent with the period of polycythemia, serum sodium values returning to normal with the normalization of hemoglobin and hematocrit. Throughout the hospitalization, the pulmonary radiological appearance was not altered. The patient was discharged in a markedly improved general condition with two negative SARS-CoV-2 RT-PCR samples. Unfortunately, given the epidemiological context, the patient could not be re-evaluated later.

The evolution of hemoglobin compared to the N/L ratio is shown in figure 8 .

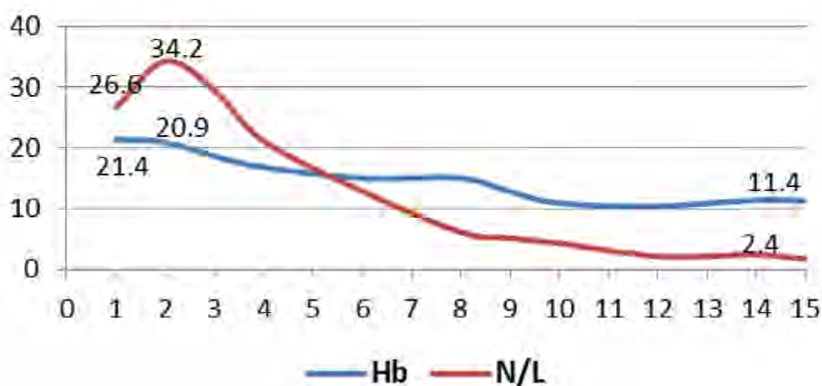

FIGURE 8. Evolution of hemoglobin values compared to neutrophil/lymphocyte ratio (patient 3 )

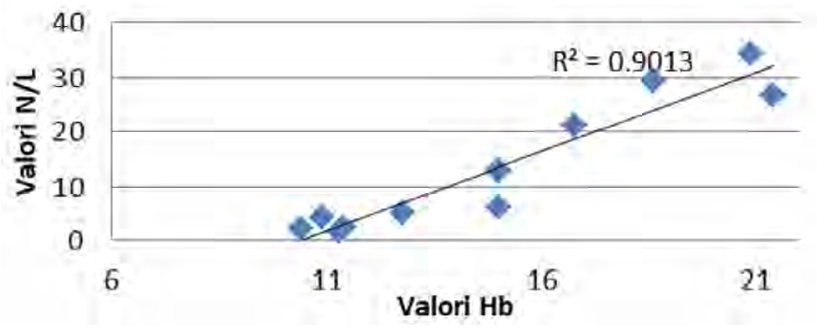

FIGURE 9. Hb correlation with $N / L$ ratio (patient 3)

Figure 9 shows the correlations of hemoglobin values with $\mathrm{N} / \mathrm{L}$ ratio values. There is a very good correlation of value pairs.

\section{DISCUSSIONS}

The case presented an important polycythemia, probably secondary, which could be put in the context of the current infection, because, with the favorable evolution of the patient, demonstrated both clinically and paraclinically, the number of erythrocytes decreased significantly. However, without a rigorous hematological evaluation, we cannot put this phenomenon in direct connection with SARS-CoV-2 infection, most likely its etiology being multifactorial. Another peculiarity of the case was hypernatremia, which persisted for several days, despite the volume replenishment and the use of the loop diuretic. Also, we did not show a respiratory damage, in this case, the manifestations of the disease being mainly hematological, hepatic, renal.

\section{CONCLUSIONS}

We presented three cases of patients from different age groups, with different evolutionary manifestations. SARS-CoV-2 infection, due to its novelty and the complexity of the phenomena that occur, requires significant efforts from clinicians to understand the clinical manifestations, in correlation with imaging and laboratory data. Each patient has particularities, which can play a central role in the clinical and evolutionary picture. The calculation of the severity score at admission had a rather low degree of sensitivity, perhaps due to the fact that patients were hospitalized on different days of the disease. The first case was hospitalized on the 3rd day of clinical evolution, was classified as medium risk and ended up in intensive care, and the other two, classified as high risk, did not require admission to ATI.

Negative molecular biology results cannot invalidate the diagnosis of COVID-19 in the presence of disease and / or epidemiological evidence.

Virological cure of patients is not necessarily a clinical cure and, therefore, a large part of patients will require further multidisciplinary follow-up, to capture a possible chronic evolution of organic damage during acute infection.

Conflict of interest: none declared Financial support: none declared 


\section{REFERENCES}

1. Rodriguez-Morales AJ, Cardona-Ospina JA, Gutiérrez-Ocampo E et al. Clinical, laboratory and imaging features of COVID-19: A systematic review and meta-analysis. Travel Med Infect Dis. 2020;34:101623.

2. Rong-Hui Du, Li-Rong Liang, Cheng-Qing Yang et al. Predictors of Mortality for Patients with COVID-19 Pneumonia Caused by SARS-CoV-2: A Prospective Cohort Study. European Respiratory Journal 2020.

3. Wenhua Liang, Hengrui Liang, Limin Ou et al. Development and Validation of a Clinical Risk Score to Predict the Occurrence of Critical Illness in Hospitalized Patients With COVID-19. JAMA Intern Med. Published online May 12, 2020.

4. https://qxmd.com/calculate/calculator_731/covid-19-prognostic-tool.
5. Ordonantă militară nr. 6 din 30 martie 2020 http://legislatie.just.ro/ Public/DetaliiDocument/224493.

6. James Hansen. Lung age is a useful concept and calculation. Primary Care Respiratory Journal. 2010; 19(4):400-401.

7. Xie X, Zhong Z, Zhao W, Zheng C, Wang F, Liu J. Chest CT for typical 2019-nCoV pneumonia: relationship to negative RT-PCR testing. Radiology. 2020.

8. Zhang W, Du RH, Li B, Zheng XS, Yang XL, Hu B. Molecular and serological investigation of 2019-nCoV infected patients: Implication of multiple shedding routes. Emerg Microbes Infect. 2020;9:386-389.

9. Terpos E, Ntanasis-Stathopoulos I, Elalamy I et al. Hematological findings and complications of COVID-19. Am J Hematol. 2020;95(7):834-847. 